\title{
Prototype Design and Experimental Test of a Rotorcraft Capable of Adhering to and Moving on the Ceiling
}

\author{
Masaya Yasunaga , Jae Hoon Lee and Shingo Okamoto \\ Graduate School of Science and Engineering, Ehime University, Japan
}

\begin{abstract}
This paper presents the prototype design and evaluation of a novel rotorcraft that can adhere to and move on the ceiling. It has four propellers for flying like conventional rotorcraft. In addition, a specialized guard frame and two active wheels are attached on its body frame for adhering to and moving on the ceiling. The propellers create negative pressure in the guard frame that acts as a suction cup for generating adhesion force. The active wheels are used for moving on the ceiling face. Experiments are carried out to show the feasibility of flying in the air, adhering to, and moving on the ceiling.
\end{abstract}

\section{Introduction}

There are about 700,000 bridges in Japan. In 2010, 50 years had elapsed since about $8 \%$ of them had been constructed. Moreover, it is expected that about $26 \%$ and $53 \%$, in 2020 and in 2030 respectively, will be considered deteriorated bridges with ages of more than 50 years [1]. Likewise, rapid deterioration of physical infrastructure, including bridges, is recognized as a big problem in Japan. Old infrastructures requires a huge maintenance cost. The safety of the worker and the worker's accessibility to the inspection area are the main difficulties in inspection tasks. Therefore, robotics technology has been attracting attention from researchers because it is recognized as a solution for these problems. Particularly, several types of multi-rotor crafts have been developed for effective inspection processes because they show high performance in mobility to access target positions [2-4]. Recently, experimental tests with unmanned aerial vehicles (UAVs) for bridge inspection have been carried out field situations [5].

The most important capability of a multi-rotor craft for inspection applications is stable hovering. Namely, the rotorcraft should have the capability of stable flight in a high place near the bridge for inspection tasks such as taking pictures. However, the fatal drawback while using a multi-rotor craft is the fact that it is sensitive to wind. Generally, it shows good performance in navigating to the goal position through free space because of excellent motion control technology with GPS sensor system. However, in high places near the structure, sometimes there are complex and strong winds, so the rotorcraft cannot keep its posture and may collide with the structure. In addition, the fundamental limitation of a UAV is that, it cannot access the ceiling surface of bridge structure or a building. For this reason, robots having the capability of adhering to the ceiling or wall has got an attention from the robotics research society [6-10]. So far, their fundamental limitation is that they can move only on the face of the wall, and cannot fly in the air. Thus, it is impossible to move directly to the task position.

To cope with the above-mentioned problems, a novel rotorcraft is presented in this research. In addition to the basic flying functions of a conventional rotorcraft, a special device to provide the capabilities for adhering to and moving on the ceiling was include in the proposed system. For this purpose, a specialized frame with active wheels was designed. Additionally, the prototype was constructed and experimental tests were carried out.

\section{Method for adhering to and moving on the ceiling}

\subsection{Adhering to the ceiling}

For a rotorcraft's adhesion function on the ceiling, several methods, such as magnetics or suction cups could be applied. However, most rotorcraft cannot carry additional heavy devices because their loading capacity is so small.

The innovative idea of this paper is to design a specialized guard frame for utilizing propellers in both the flying and adhering objectives as shown in Fig. 1. Using lightweight material for the guard is very effective in reducing the weight of the device, while providing additional functionality. Propellers are used for flying in the air in the same way as other rotorcraft. At the same time, by installing a special guard frame around the propeller, an enclosed space is formed between the ceiling surface and the propeller while adhering. Moreover, the propeller expels the air in the enclosed 
space, creating a negative pressure in the space. Resultantly, the propeller guard helps form a vacuum.

\subsection{Moving on the ceiling}

While adhering on the ceiling, the propellers cannot generate moving force in the horizontal direction because the lift forces of the propellers act vertically. In order to move the rotorcraft, two active wheels that can be controlled independently were installed on the platform. By changing the velocities of both wheels, translational and rotational motions of the system can be achieved. A set of more than three omni-directional wheels could be used for generating moving force. In that case, the rotorcraft could move on the ceiling surface omnidirectionally; i.e., both translational and rotational motions could be achieved independently. However, it would require more than three wheels and motors, with their corresponding extra weight. Therefore, two active wheels were employed in this research.

The forces acting on the rotorcraft while it moves on the ceiling are depicted in Fig. 2. The rotation of the propeller generates the lift force, $F_{\text {thrust }}$, which compensates for the rotorcraft's weight, $F_{\text {gravity }}$, and lifts up the system during both flying and attaching. The suction force, $F_{\text {vacuum }}$, makes the system attach to the ceiling face, and becomes zero during flying motion. By rotating the wheels while attaching, the moving force $F_{\text {traction }}$ is generated. When it is larger than the friction force between the ceiling face and the guard, $F_{\text {friction }}$, the system can move.

\section{Development of rotorcraft prototype}

In order to examine the feasibility of the proposed design, a four-rotor craft prototype has been constructed.

\subsection{Platform design}

The structure of a well-known quad-copter with four propellers was employed as the basic platform design. A ready-made carbon frame of IRON MAN 650 made by TAROT Co. was used for the system. Motors and propellers were selected that can provide sufficient payload capacity for additional devices including the special guard and inspection instrument. Two servomotors were used to move the system by rotating the wheels on the ceiling. A 6-cell lithium-polymer battery was embedded in the platform as the power source. The total mass of the aircraft prototype, including the battery is about $3.3 \mathrm{~kg}$. The four-rotor craft prototype is displayed in Fig. 3.

\subsection{Special guard frame for generating suction force}

The proposed special guard was designed to enable the functions of adhering to and moving on the ceiling by creating a vacuum. It was made with lightweight
Styrofoam and attached to the platform. The radical gap between each propeller edge and its guard was made as small as possible to maximize the effects of negative pressure inside the guard itself. In the prototype, this gap was set to about $1 \mathrm{~cm}$. Figure 3 shows the guard, made of white Styrofoam board, constructed for this study. Additionally, a flexible suction plate, with a semi-circular cross section, was installed on the upper surface of the guard to increase sealing performance and reduce the friction with the ceiling surface. It was made with PET plate in this research. Moreover, it helps maintain the suction force on uneven surfaces. The prototype is 940 $\mathrm{mm}$ wide, $940 \mathrm{~mm}$ of long, and $300 \mathrm{~mm}$ of high.

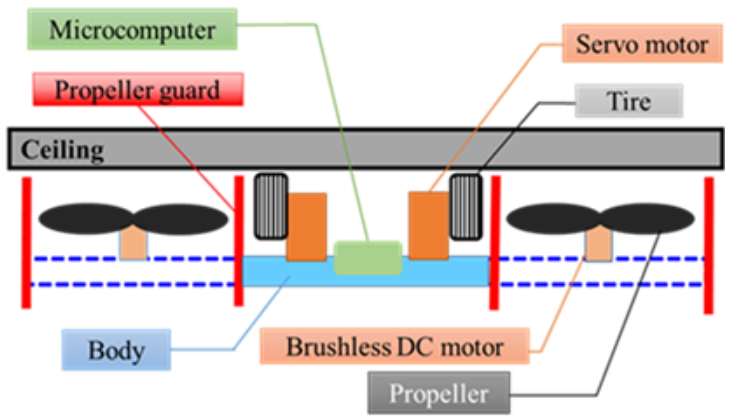

Figure 1. Schematic diagram of the rotorcraft prototype

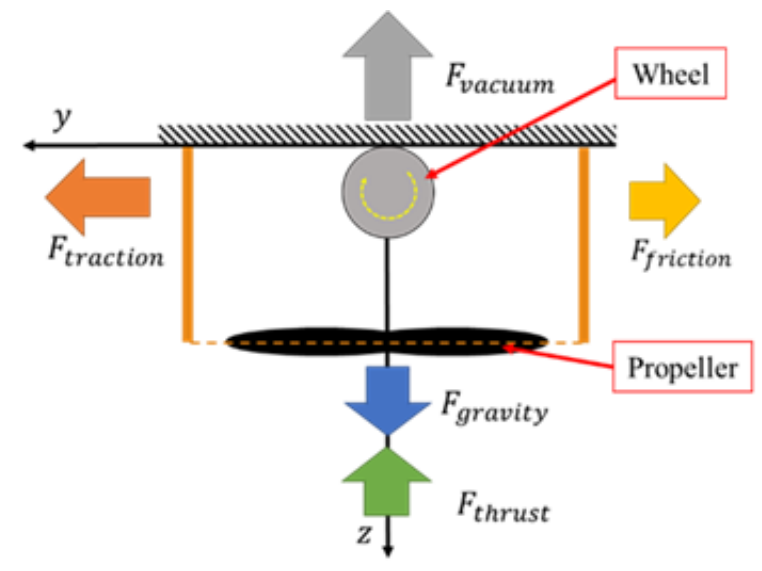

Figure 2. Schematic diagram of the forces acting on the rotorcraft while moving on the ceiling

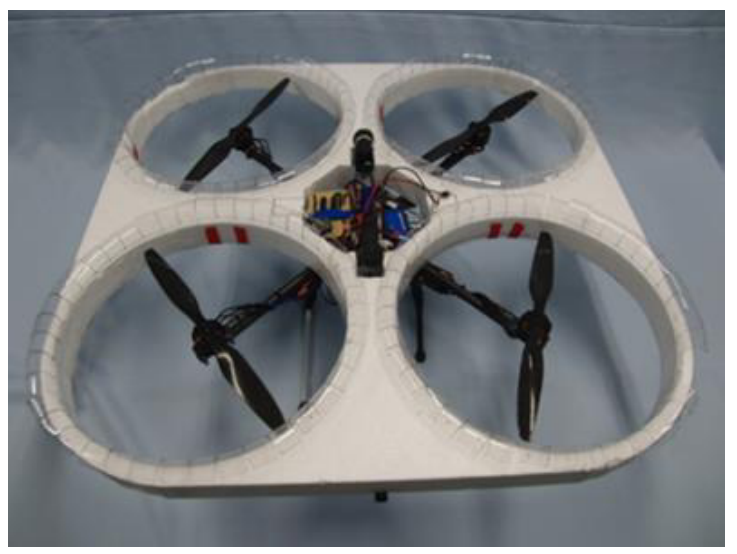

Figure 3. The rotorcraft prototype developed in this research 


\subsection{Controller configuration in the developed prototype}

Figure 4 shows the schematic diagram of the controller configuration in the developed four-rotor craft. It consists of an embedded controller with a receiver, four propellerdriver sets, two servo motors for active wheels, a battery, a computer for data monitoring, and a transmitter for user interface. The embedded controller is connected to the computer and the transmitter with Bluetooth wireless communications. Operating commands are sent from the transmitter to the controller, and include reference velocities for rotational and vertical motion. Then, the controller translates them into rotational velocities for each propeller, and sends them to each motor driver. In addition, basic attitude control was included in the embedded controller; thus, it is possible to stably hover even without input from the transmitter. The computer is used to record information such as posture angles and operating commands.

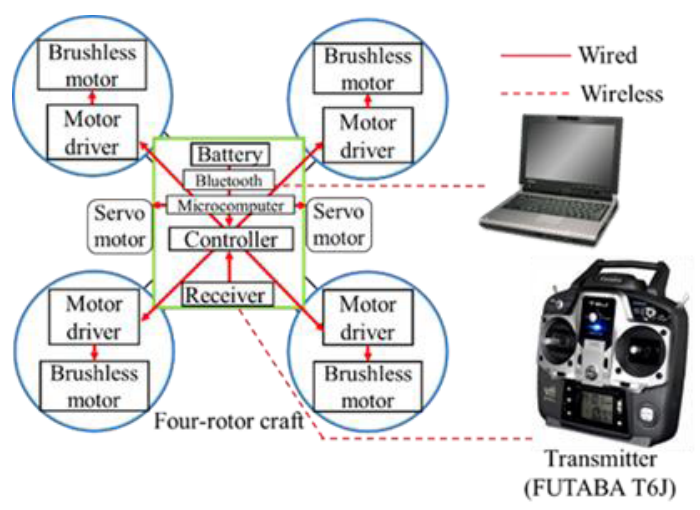

Figure 4. Configuration of the control system of the developed rotorcraft

\section{Experimental tests}

Experimental studies were carried out to verify the characteristics of the rotorcraft prototype developed in this research.

\subsection{Method for experiments}

The basic function, flying in the air, and the additional functions, adhering to and moving on the ceiling, were demonstrated with the developed rotorcraft prototype. All motion was generated by manual operation of the transmitter. The experimental motion scenario consists of taking off, hovering in the air, moving to the ceiling, moving on the ceiling, and landing. The experiments were conducted in an indoor environment. The ceiling surface has a decorative of board whose irregularity of about $1 \mathrm{~cm}$ in height.

Two designs, with the propeller inside of or below the guard, were constructed, in order to check their suction performance. In the A-type prototype, each propeller is installed inside of the suction plate as shown in Fig. 5.

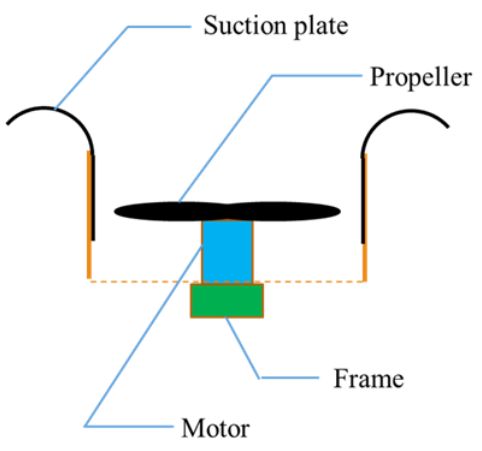

(a) Schematic diagram of A-type prototype

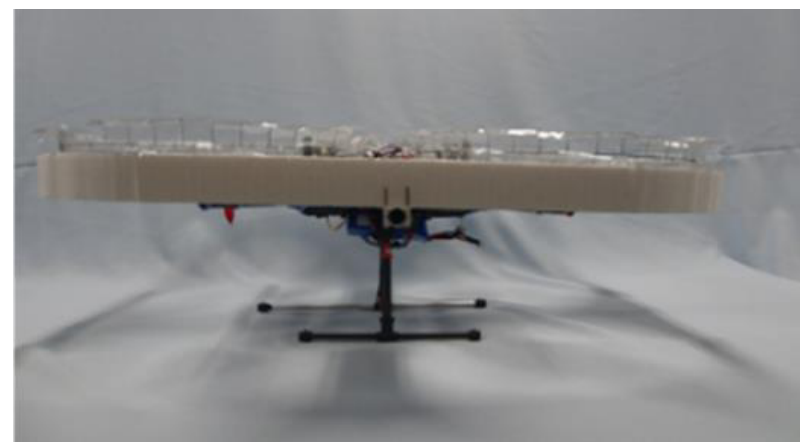

(b) Side view of A-type prototype

Figure 5. Prototype rotorcraft of A-type: Propellers are installed inside of suction plate

On the other hand, the propellers are installed below the suction plate in the B-type as shown in Fig. 6. Both prototypes were subjected to the same experimental scenario and results were compared.

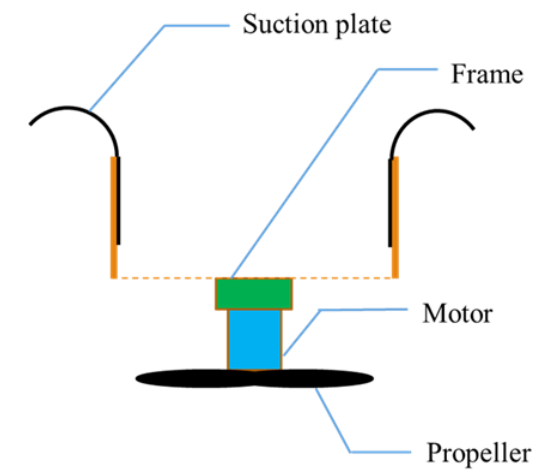

(a) Schematic diagram of B-type prototype

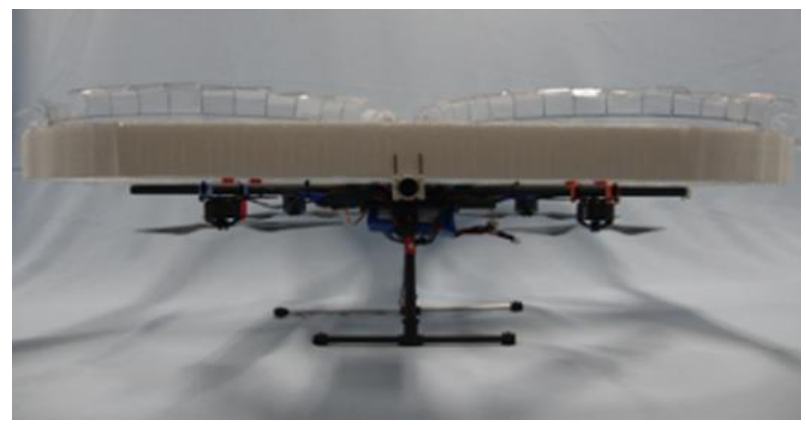

(b) Side view of B-type prototype

Figure 6. Prototype rotorcraft of B-type: Propellers are attached below the suction plate 


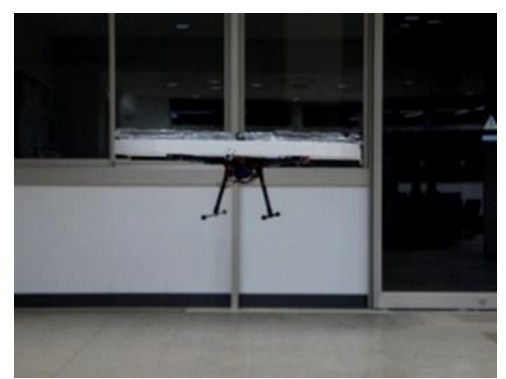

(a)

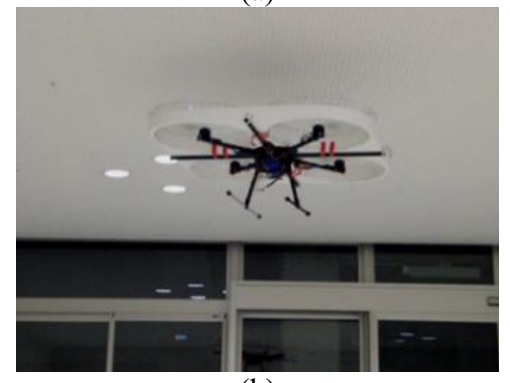

(b)

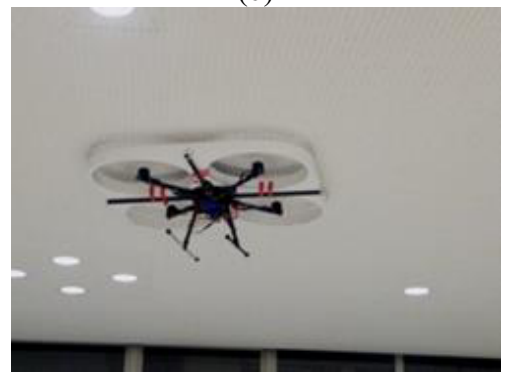

(c)

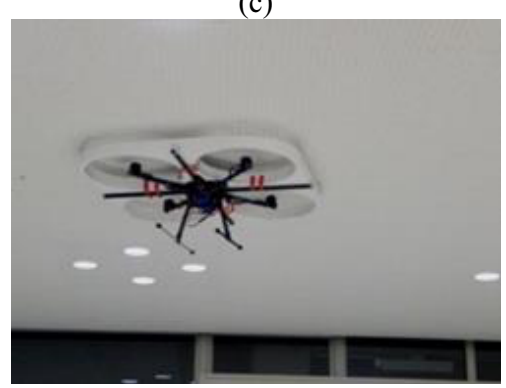

(d)

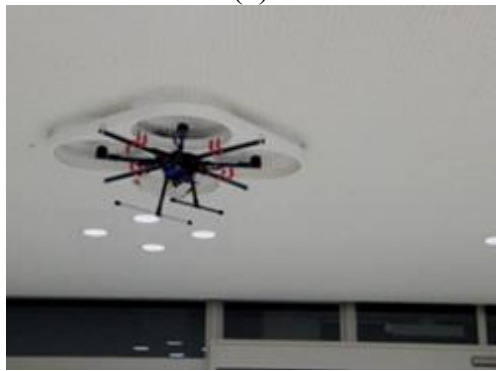

(e)

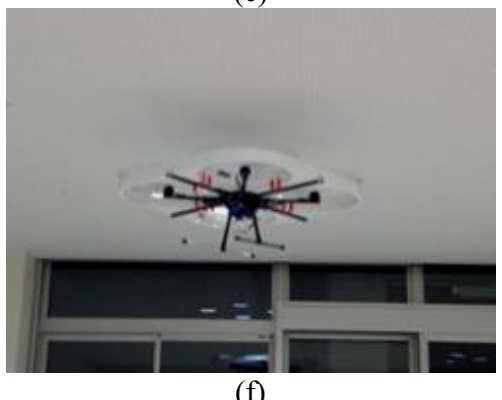

(f)

Figure 7. Experimental result by using the A-type rotorcraft

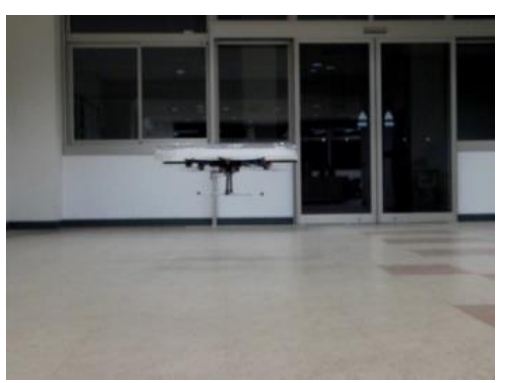

(a)

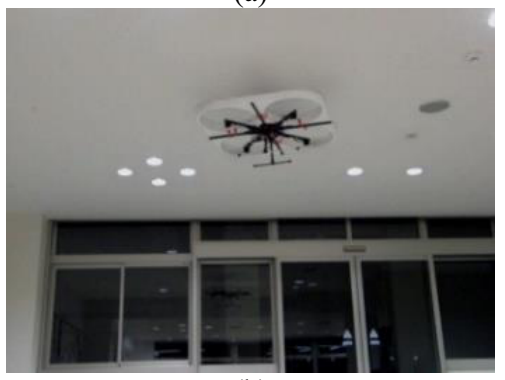

(b)

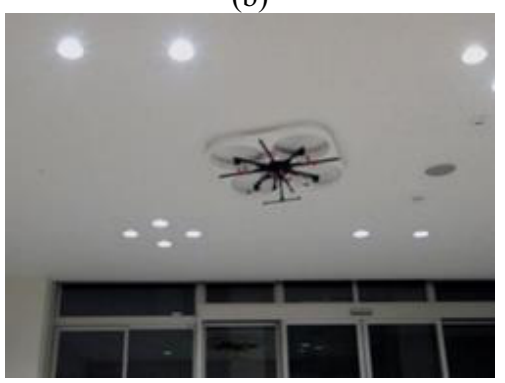

(c)

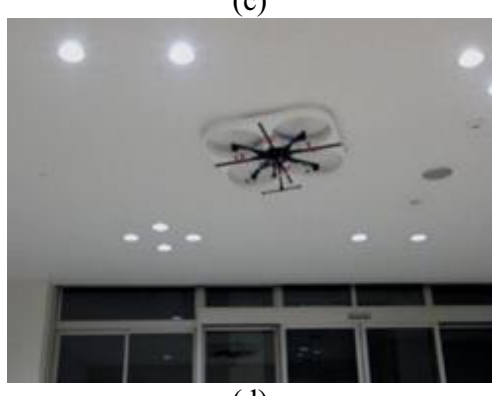

(d)

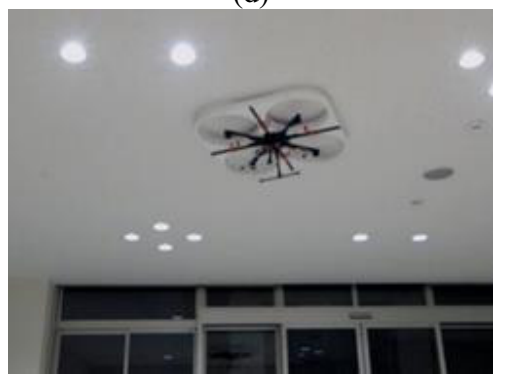

(e)

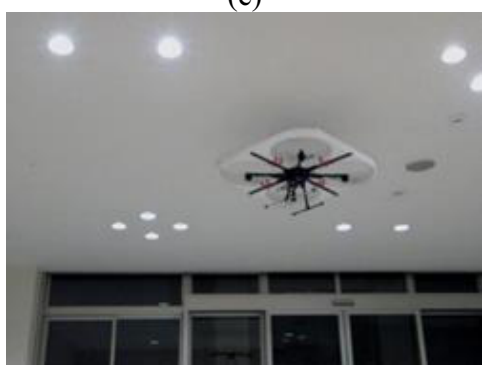

(f)

Figure 8. Experimental result by using the B-type rotorcraft 


\subsection{Experimental results}

Figures 7 and 8 show the scenes of experiments with Atype and B-type prototypes. It was possible to fly in the air for both cases. In addition, both prototypes could move on the ceiling while adhering.

It was observed that the performance of each motion type was different four each prototype. The B-type has better flying capability than the A-type because its propellers are installed outside of the suction plate and can generate a lager lift force. Conversely, for adhering motion, the A-type rotorcraft shows better performance than the B-type. The required power for both kinds of motion, i.e., hovering and adhering to the ceiling, was measured experimentally in this research. The differences between the prototype are outlined in Table 1. For a hovering motion, the required motor power for the B-type body is $7 \%$ smaller than for the A-type. In contrast to this, when the prototype is adhering to the ceiling, the required motor power for the A-type body is $11 \%$ smaller than for the B-type.

Table 1. Motor power required for each motion with two type of rotorcraft prototype

\begin{tabular}{|c|c|c|}
\hline \multirow{2}{*}{ Type of rotor craft } & \multicolumn{2}{|c|}{ Motion of the rotorcraft } \\
\cline { 2 - 3 } & Hovering & Adhering to the ceiling \\
\hline A-Type & $47 \%$ & $22 \%$ \\
\hline B-Type & $40 \%$ & $33 \%$ \\
\hline
\end{tabular}

\section{Conclusion and future works}

A novel rotorcraft design for inspecting high-rise infrastructure was developed in this study. Two prototypes were proposed and developed. The creative idea to utilize the propeller for both flying and attaching on the ceiling was realized by manufacturing a special guard. Through experiments with the developed system, it was verified that the proposed design is very effective and energy saving for attaching and moving on the ceiling surface.

In this study, we carried out experiments where a four-rotor craft adhered not only to a smooth surface but also a surface with irregularities in width and height of about $1 \mathrm{~cm}$. However, the bottom surface of an old bridge is likely to be a more complex surface. Therefore, future challenges include coping with irregular surfaces, and designing the suction plate to be more flexible to ensure the suction force. Currently, research on autonomous flight control and outdoor field inspection trials are ongoing.

\section{Acknowledgements}

This research was supported by Okumuragumi Research Fund.

\section{References}

1. Ministry of Land, Infrastructure, Transport and Tourism, The maintenance of national road network in Japan, (2009)

(http://www.mlit.go.jp/road/road_e/03key_challenge s/1-2-2.pdf)

2. P. Liu, A.Y. Chen, Y.-N. Huang, J.-Y. Han, J.-S. Lai, S.-C. Kang, T.-H. Wu, M.-C. Wen, and M.-H. Tsai, A Review of Rotorcraft Unmanned Aerial Vehicle Developments and Applications in Civil Engineering, Smart Structures and Systems, 13, 6 (2014)

3. L. Wallace, A. Lucieer, C. Watson, and D. Turner, Development of a UAV-LiDAR System with Application to Forest Inventory, Remote Sensing, 4, 6(2012)

4. C. Deng, S. Wang, Z. Huang, Z. Tan, and J. Liu, Unmanned Aerial Vehicles for Power Line Inspection: A cooperative Way in Platforms and Communications, Journal of Communications, 9, 9 (2014).

5. Barritte Lovelace, Principal Investigator Collins Engineers Inc., Unmanned Aerial Vehicle Bridge Inspection Demonstration Project, Research Project Final Report (2015).

6. I.-G. Koo, T.-D. Trong, Y.-H. Lee, H. Moon, J. Koo, S.-K. Park, H.-R. Choi, Development of Wall Climbing Robot System by Using Impeller Type Adhesion Mechanism, journal of intelligent and Robotic System, 57-72, (2013).

7. Mahmoud Tavakoli, C. Viegas, L. Marques, J. N. Pires, A. T. de Almedia, OmniClimbers: Omnidirectional magnetic wheeled climbing robots for inspection of ferromagnetic structures, Robotics ad Autonomous System, 61,997-1007,(2013).

8. D. Schmidt, C. Hillenbrand and K. Berns, Omnidirectional locomotion and traction control of the wheel-driven, wallclimbing robot, Robotica, 29,991-1003,(2011).

9. Kiyoshi Tsuru, S. Hirose, Development of Vmax III: Magnetic Wall Climbing Robot with Holonomic and Omni-directional Mobility, Journal of the Robotics Society of Japan ,30, 639-647,(2012).

10. J.C. Grieco, M. Prieto, M. Armada, P. Gonzales de Santos, A Six-Legged Climbing Robot for High Payloads, Proceeding of the 1998 IEEE International Conference on Control Applications, 1, 446450,(1998). 